\title{
FLORESCIMENTO DE TANGERINEIRAS SATSUMA 'OWARI' TRATADAS COM PACLOBUTRAZOL, ANELAMENTO DO CAULE E BAIXA TEMPERATURA ${ }^{1}$
}

\author{
DALMO LOPES DE SIQUEIRA², JOSÉ LUIS GUARDIOLA BARCENA, MARLON DUTRA DEGLI ESPOSTI ${ }^{4}$
}

\begin{abstract}
RESUMO - O retardador de crescimento paclobutrazol (PBZ) e o anelamento dos ramos foram aplicados em mudas de tangerineira satsuma 'Owari' sobre porta-enxerto de citrange 'Carrizo', cultivadas em vaso e mantidas em dois ambientes (câmaras de crescimento com temperatura diurna de $15^{\circ} \mathrm{C}$ e noturna de $8^{\circ} \mathrm{C}$ e com temperatura diurna de $26^{\circ} \mathrm{C}$ e noturna de $20^{\circ} \mathrm{C}$ ). Houve aumento significativo no florescimento da tangerineira 'Satsuma', devido ao regime de baixas temperaturas $\left(15 / 8^{\circ} \mathrm{C}\right)$; entretanto, o PBZ não foi efetivo na indução do florescimento da tangerineira 'Satsuma' em ambas as condições de temperatura $\left(15 / 8^{\circ} \mathrm{C}\right.$ e $\left.26 / 20^{\circ} \mathrm{C}\right)$. O florescimento aumentou quando as plantas foram aneladas, principalmente nas condições de temperaturas baixas $\left(15 / 8^{\circ} \mathrm{C}\right)$. A aplicação de paclobutrazol e o anelamento induziram variações nas concentrações de carboidratos nas folhas e raízes das plantas; entretanto, não foi possível estabelecer relação de causa-efeito entre florescimento e os níveis de carboidratos na planta.
\end{abstract}

Termos para indexação: retardador de crescimento, carboidratos, indução floral

\section{FLOWERING OF SATSUMA 'OWARI’ MANDARINS TREATED WITH PACLOBUTRAZOL, STEM GIRDLING AND LOW TEMPERATURE}

\begin{abstract}
The growth retardant paclobutrazol (PBZ) and stem girdling were applied to satsuma 'Owari' mandarin plants cultivated in pots in a growth chamber under two different environmental conditions: one with temperature at day time set in $15^{\circ} \mathrm{C}$ and night time in $8^{\circ} \mathrm{C}$, and another with temperature at day time set in $26^{\circ} \mathrm{C}$ and night time in $20^{\circ} \mathrm{C}$. The low temperature $\left(15 / 8^{\circ} \mathrm{C}\right)$ significantly increased flowering, but $\mathrm{PBZ}$ was not effective. The flowering increased when the plants were girdled, mainly in the conditions of low temperatures $\left(15 / 8^{\circ} \mathrm{C}\right)$. Paclobutrazol and the girdling induced variations in carbohydrates concentrations of leaf and root, however it was not possible to establish a relationship between flowering and carbohydrates level in the plant.
\end{abstract}

Index terms: growth retardant, carbohydrates, floral induction

\section{INTRODUÇÃO}

Paclobutrazol (PBZ) e uniconazol (UNI) são os triazóis mais efetivos na redução do crescimento de plantas (Graebe, 1987; Rademacher, 1997; Fletcher et al., 2000). De modo geral, as pesquisas realizadas com esses produtos em citros apresentam como objetivo, reduzir o crescimento das plantas e promover o florescimento.

O PBZ é efetivo no florescimento de diversas variedades e espécies de citros (Snowball et al., 1994; Iwahori \& Tominaga, 1986; Ogata et al., 1995; Okuda et al., 1996; Yamashita et al., 1997; Delgado et al., 1995; Harty \& Van Staden, 1988; Acosta et al., 1994; Delgado et al., 1986); entretanto, parecem existir diferenças entre variedades na efetividade dos retardadores de crescimento. Davenport $(1986 ; 1987)$ não conseguiu obter florescimento da lima ácida Tahiti (Citrus latifolia), com paclobutrazol e uniconazol, em experimentos de campo e casa de vegetação, usando aplicações no solo e foliar. Snowball et al., (1994) também não obtiveram florescimento no pomelo 'Marsh seedless' (Citrus paradisi), com a aplicação de paclobutrazol, enquanto a lima ácida ‘West Indian' e cunquate 'Meiwa' floresceram abundantemente nas mesmas condições.

A aplicação de paclobutrazol, além de alterar características fenotípicas da planta (florescimento, encurtamento dos entrenós), também pode modificar concentração de carboidratos na planta, bem como a relação carboidratos na parte aérea/raízes (Mehouachi et al., 1996; Okuda et al., 1996). Estas modificações podem ser responsáveis pelas alterações fenotípicas mencionadas, embora seja difícil estabelecer relação de causaefeito entre carboidratos e crescimento e desenvolvimento de plantas

Quanto à época de aplicação, Davenport (1990) cita que não existem relatos sobre a efetividade do paclobutrazol em induzir o florescimento dos citros, quando aplicado em períodos em que as condições ambientais não são indutivas ao florescimento.

Segundo Goldschmidt et al. (1970), as giberelinas endógenas são importantes para o florescimento dos citros, pois causam a sua inibição. O florescimento ocorre quando condições ambientais inadequadas ao crescimento das plantas (baixas temperaturas ou estresse hídrico) interrompem o crescimento das raízes e, conseqüentemente, a síntese de giberelinas.

Assim sendo, a aplicação de retardadores (inibidores da síntese de giberelinas) deveria ser capaz de promover o florescimento em qualquer época do ano, fato que não está comprovado até o momento (Davenport, 1990). A possibilidade de obter o florescimento dos citros em qualquer época do ano, com o uso de retardadores, apresenta importância prática devido aos melhores preços alcançados pelos frutos produzidos na entressafra.

O esclarecimento do papel dos fatores ambientais sobre o controle do florescimento somente é possível com a realização de experimentos em ambiente controlado (Spiegel-Roy \& Goldschmidt, 1996); entretanto, em experimentos realizados nessas condições, freqüentemente, há necessidade de se trabalhar com um número reduzido de plantas em função da disponibilidade limitada de espaço.

O presente trabalho foi realizado com o objetivo de estudar a atuação do paclobutrazol no crescimento e florescimento da tangerineira Satsuma 'Owari', quando aplicado em plantas induzidas e não induzidas ao florescimento e as possíveis relações entre acúmulo de carboidratos, florescimento e aplicação de paclobutrazol.

\section{MATERIALEMÉTODOS}

Os experimentos foram realizados na Universidade Politécnica de Valência - Espanha, cuja implantação ocorreu no dia 07-11-2000, utilizando mudas de tangerineira satsuma 'Owari' enxertadas sobre citrange 'Carrizo' [(Citrus sinensis (L.) Osbeck x Poncirus trifoliata (L.) Raf.)], com idade aproximada de 1,5 ano. As plantas foram cultivadas em vasos contendo 05 litros de substrato. As mudas foram mantidas em casa de vegetação até o início dos experimentos, sendo regularmente irrigadas e adubadas com solução N, P, K, Mg. e micronutrientes.

Foram realizados dois experimentos simultaneamente, utilizando a mesma metodologia, com exceção da temperatura ambiental da câmara de crescimento. Doravante, os dois experimentos serão denominados "experimento $15 / 8$ " e "experimento 26/20".

Utilizou-se o delineamento inteiramento casualizado, em esquema

\footnotetext{
1 (Trabalho 048/2004). Recebido: 29/04/2004. Aceito para publicação: 19/10/2004

${ }^{2}$ Eng. Agr., Dr., Professor do Departamento de Fitotecnia, Universidade Federal de Viçosa - CEP 36570-000 - Viçosa - MG - email - siqueira@ ufv.br.

${ }_{3}^{3}$ Professor Catedrático do Departamento de Biologia Vegetal da Universidade Politécnica de Valencia - Espanha - email - jlguardiola@bvg.upv.es

${ }^{4}$ Eng. Agr., Doutorando em Fitotecnia - Universidade Federal de Viçosa - CEP 36570-000 - Viçosa - MG - email: mesposti@ bol.com.br.
} 
fatorial 2 × 2, cujos fatores foram aplicação de paclobutrazol (com e sem) e anelamento (com e sem), totalizando 04 tratamentos, com cinco repetições e uma planta por parcela.

O paclobutrazol foi aplicado no solo, na concentração de 1.000 $\mathrm{mg} /$ planta de ingrediente ativo, utilizando o produto comercial Cultar $(250 \mathrm{~g} /$ litro), que foi dissolvido em $300 \mathrm{ml}$ de água e uniformemente aplicado na superfície dos vasos.

O anelamento foi realizado na circunferência do caule das plantas, a $5 \mathrm{~cm}$ acima do local da enxertia, sem remover porções da casca e tomandose o cuidado de não aprofundar a ferramenta no lenho das plantas para não danificar o xilema.

No experimento 15/8, as plantas foram submetidas à indução floral, colocando-as em câmara de crescimento, com fotoperíodo de $16 / 8$ horas (luz/escuro) e com temperatura diurna de $15^{\circ} \mathrm{C}$ e noturna de $8^{\circ} \mathrm{C}$, onde permaneceram por 60 dias.

No experimento 26/20, as plantas também foram mantidas em câmara de crescimento, porém em temperaturas de $26^{\circ} \mathrm{C} / 20^{\circ} \mathrm{C}$ (dia/noite), para forçar a brotação, com fotoperíodo de 16/8 horas (luz/escuro).

Foi realizado, para cada tratamento, uma avaliação inicial de açúcares redutores pelo método de Park \& Johnson (1949), de açúcares totais e amido pelo método antrona-ácido sulfúrico (McCready et al., 1950), nas folhas e nas raízes. Foi usada uma folha por planta e raízes, com diâmetro compreendido entre 0,5 a $2,0 \mathrm{~mm}$ (Tabela 1 ).

Aproximadamente 30 dias e 60 dias após o início dos experimentos (11-12-2000 e 17-01-2001), a análise de carboidratos foi repetida para as plantas do experimento 15/8. Neste caso, foram usadas duas repetições, sendo uma parcela constituída por 03 plantas e outra por duas plantas.

No dia 13-12-2000, foi avaliada a percentagem de nós brotados, separadamente para cada surto de brotação. Quando as avaliações foram realizadas, as plantas apresentavam apenas 03 surtos de crescimento, sendo que o primeiro, originário da haste principal e responsável pela formação das "pernadas" da planta, foi denominado surto 1. Os ramos desenvolvidos a partir das brotações do surto 01 foram denominadas surto 2, e as brotações apicais, originárias do surto 2, foram denominadas de surto 3. A avaliação foi feita somente nas plantas do experimento 26/20, pois as demais ainda não haviam começado a brotar. A avaliação foi repetida em 04-01, quando as plantas já haviam terminado a emissão de brotos.

No dia 07-02-2001, quando as plantas do experimento $15 / 8$ se encontravam em pleno florescimento, foram avaliados o número de flores em cada surto de crescimento, conforme mencionado anteriormente, e calculado o número de flores por 100 nós.

As análises estatísticas foram efetuadas separadamente para cada experimento. Não foi possível realizar a análise conjunta dos experimentos (Figura 1) devido ao escasso florescimento das plantas do experimento 26/20.

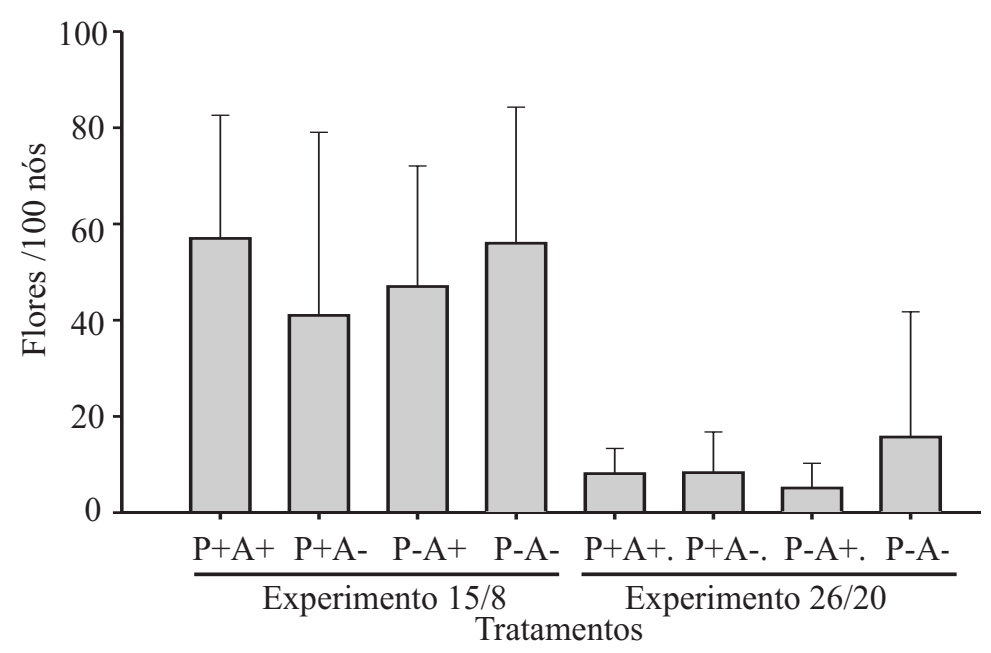

FIGURA 1 - Florescimento de tangerineiras satsuma 'Owari' tratadas com paclobutrazol e aneladas no caule (dois experimentos). $\mathrm{P}^{+}=$presença de $\mathrm{PBZ}, \mathrm{A}^{+}=$com anelamento; $\mathrm{P}^{-}=$ausência de PBZ e $\mathrm{A}^{-}=$ausência de anelamento (as barras sobre as colunas indicam o desvio-padrão).

\section{RESULTADOSEDISCUSSÃO}

A aplicação de PBZ e os anelamentos efetuados na tangerineira satsuma 'Owari' praticamente não influenciaram no florescimento; entretanto, quando as plantas foram submetidas a regimes diurnos e noturnos de temperaturas mais baixas (experimento 15/8), elas apresentaram florescimento abundante (Figura 1).

Considerando a média do número de flores obtidas para os tratamentos, de cada experimento, verificou-se que as plantas do experimento $15 / 8$ apresentaram cerca de 5,4 vezes mais flores do que as plantas do experimento 26/20, correspondendo aos valores médios de 50 e 9,3 flores/100 nós, respectivamente.

A influência de temperaturas baixas sobre o florescimento dos citros está bem documentada na literatura, sendo clássico o trabalho de MOSS (1969), que submeteu laranjeiras 'Washington Navel' a baixas temperaturas $\left(15^{\circ} / 10^{\circ} \mathrm{C}\right)$, observando acréscimo de $92 \%$ no número de flores, quando comparadas com plantas cultivadas em condições de temperaturas mais elevadas $\left(27^{\circ} / 22^{\circ} \mathrm{C}\right)$.

Os citros, para florescerem, necessitam de um período de repouso, o qual é caracterizado pela ausência ou baixo crescimento vegetativo e acúmulo de reservas nos tecidos, principalmente carboidratos. O período de repouso pode ser induzido pelo déficit hídrico ou por baixas temperaturas, sendo que a importância dos fatores mencionados depende das condições climáticas da região onde as plantas são cultivadas (Davies \& Albrigo, 1994).

As plantas produziram flores em todos os ramos, entretanto houve maior concentração na parte superior (surto 3), que apresentou valor de 61 flores $/ 100$ nós, diferindo estatisticamente $(\mathrm{P}<0,01)$ dos demais surtos (S2 e S1), que apresentaram valores de 41 e 23 flores/100 nós (Figura 2). O maior florescimento na parte superior dos ramos deve-se à característica apresentada pelos citros de florescerem em ramos com idade inferior a um ano, provenientes da brotação da primavera ou verão anterior (García-Luis \& Kanduser, 1995). Porém o trabalho foi realizado com plantas novas, que apresentam a característica de florescerem em ramos de diferentes idades, entretanto mantêm a tendência de florescimento nos surtos apicais ( mais novos).

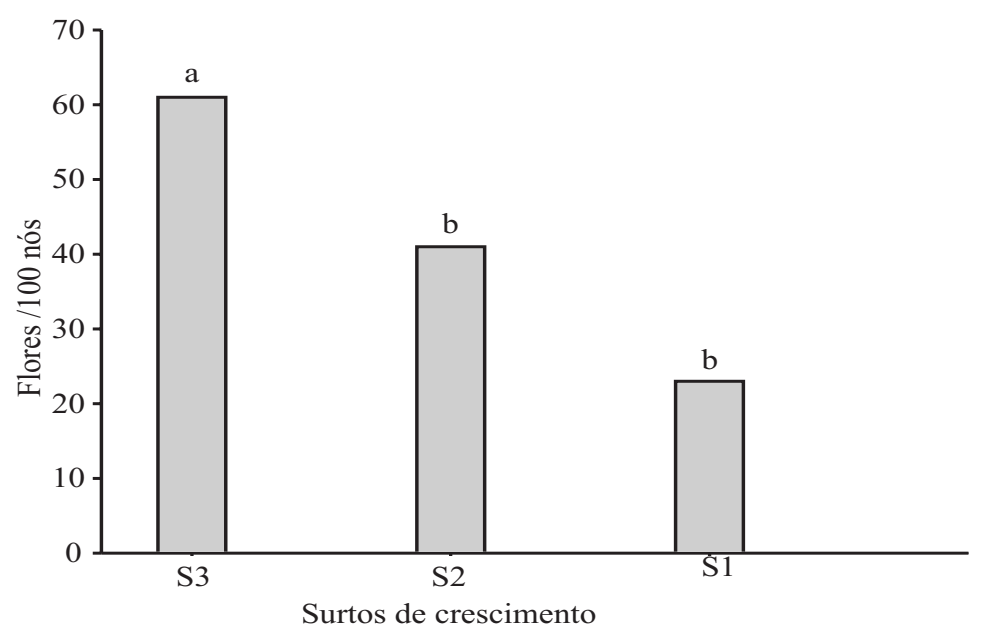

FIGURA 2 - Florescimento da tangerineira satsuma 'Owari' em três diferentes surtos de brotação (dados referentes às médias das plantas do experimento 15/8).

Considerando os efeitos individuais dos tratamentos, observase que não houve efeito da aplicação de PBZ e do anelamento (Figura 3). A ausência do efeito do PBZ sobre o florescimento das plantas (nos dois experimentos) está em desacordo com os resultados obtidos por vários autores (Iwahori \& Tominaga, 1986; Snowball et al., 1994; Ogata et al., 1995; Okuda et al., 1996; Yamashita et al., 1997; Delgado et al., 1995; Harty \& Van Staden, 1988; Acosta et al., 1994; Delgado et al., 1986), com diferentes espécies de citros. Todos os autores citados observaram aumento do número de flores nas plantas tratadas com PBZ. 


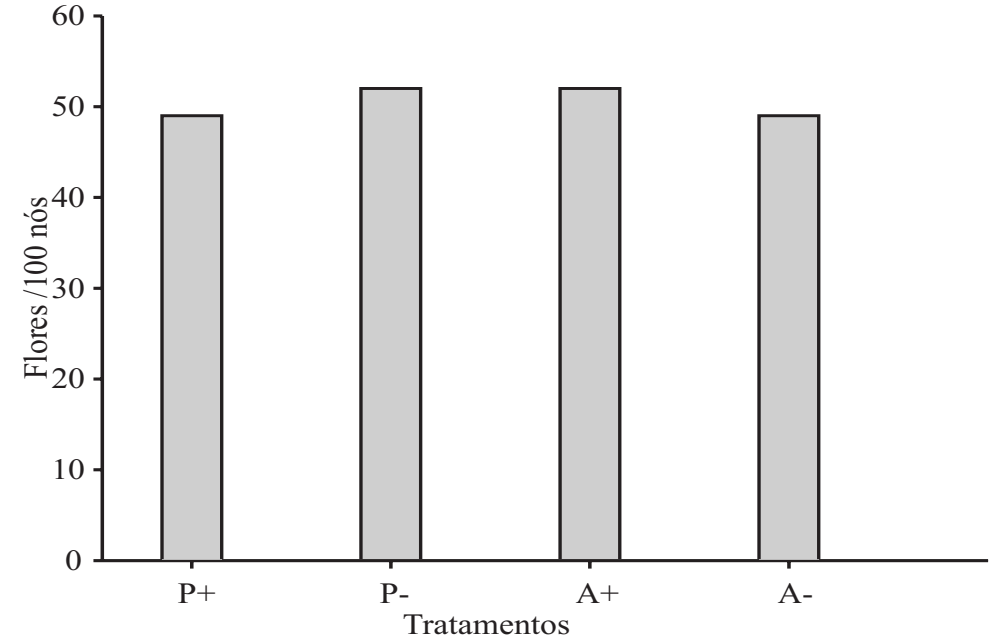

FIGURA 3 - Florescimento da tangerineira satsuma 'Owari', considerandose os efeitos individuais dos tratamentos com PBZ e anelamento do caule das plantas (dados referentes ao ambiente indutor - experimento 15/8).

Entretanto, os resultados desse trabalho estão de acordo com os obtidos por Davenport $(1986 ; 1987)$, que não conseguiu obter florescimento da lima ácida Tahiti (Citrus latifolia), aplicando PBZ no solo e foliar. Snowball et al., (1994) também não observaram florescimento no pomelo 'Marsh seedless' (Citrus paradisi), com a aplicação de PBZ, enquanto a lima ácida 'West Indian' e a cunquate 'Meiwa' floresceram abundantemente nas mesmas condições.

A ausência de efeito do PBZ em induzir o florescimento da tangerineira satsuma 'Owari' pode ser atribuída a alguns fatores, tais como a constituição genética da planta, a concentração de PBZ usada, forma de aplicação (solo ou foliar) e época de aplicação do PBZ. Entretanto, não foi possível identificar quais, entre os fatores, citados anteriormente, foram os responsáveis pela ausência do efeito sobre as plantas.
Não foram observadas diferenças nas concentrações de carboidratos nas folhas e raízes da tangerineira satsuma 'Owari' devido à aplicação de paclobutrazol e anelamento do caule.

Nas folhas, as concentrações de açúcares solúveis totais, açúcares redutores e amido apresentaram comportamento semelhante para os diferentes tratamentos ao longo do período de avaliação (Figura 4 - F). Houve tendência de acúmulo de açúcares, ao longo do tempo, devido às baixas temperaturas. Quando a época do florescimento foi se aproximando, observou-se redução nas concentrações de açúcares solúveis totais e amido nas folhas.

As concentrações de açúcares solúveis totais nas folhas apresentaram aumento médio de $44,4 \%$ no mês de dezembro, com subseqüiente queda na data posterior (jan/03). As concentrações foliares de açúcares redutores aumentaram no período (nov/02 a jan/03), com destaque para as plantas que foram aneladas e não receberam aplicações de paclobutrazol. As concentrações foliares de amido apresentaram, em média, aumento de $70,6 \%$ no período de novembro de 2002 a dezembro de 2002, mantendo-se constantes a partir desta data.

As concentrações de açúcares solúveis totais, açúcares redutores e amido nas raízes da tangerineira 'Satsuma' apresentaram variações em função dos tratamentos, sobretudo para as concentrações de amido.

Houve redução de 19,0\% nas concentrações de açúcares solúveis totais nas raízes, no período de novembro de 2002 a janeiro de 2003, sendo observado o mesmo comportamento para os demais tratamentos. As concentrações de açúcares redutores se mantiveram constantes no período de novembro de 2002 a janeiro de 2003 com uma pequena queda nas plantas que foram pulverizadas com PBZ e não aneladas.

Com relação às concentrações de amido nas raízes, observouse aumento nas plantas não aneladas e redução nas plantas aneladas (Figura 4R). Esse resultado pode ser atribuído à redução no transporte de carboidratos da parte aérea para o sistema radicular, principalmente de sacarose, que seria armazenada na forma de amido nas raízes. Além disso, as plantas aneladas apresentaram maior florescimento (Figura 3)
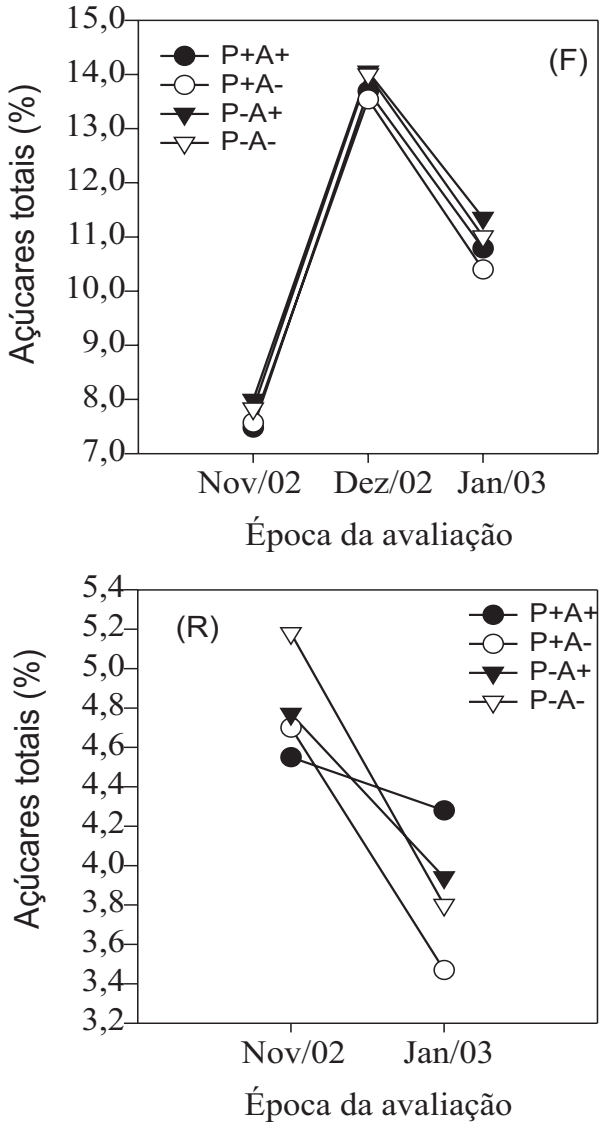
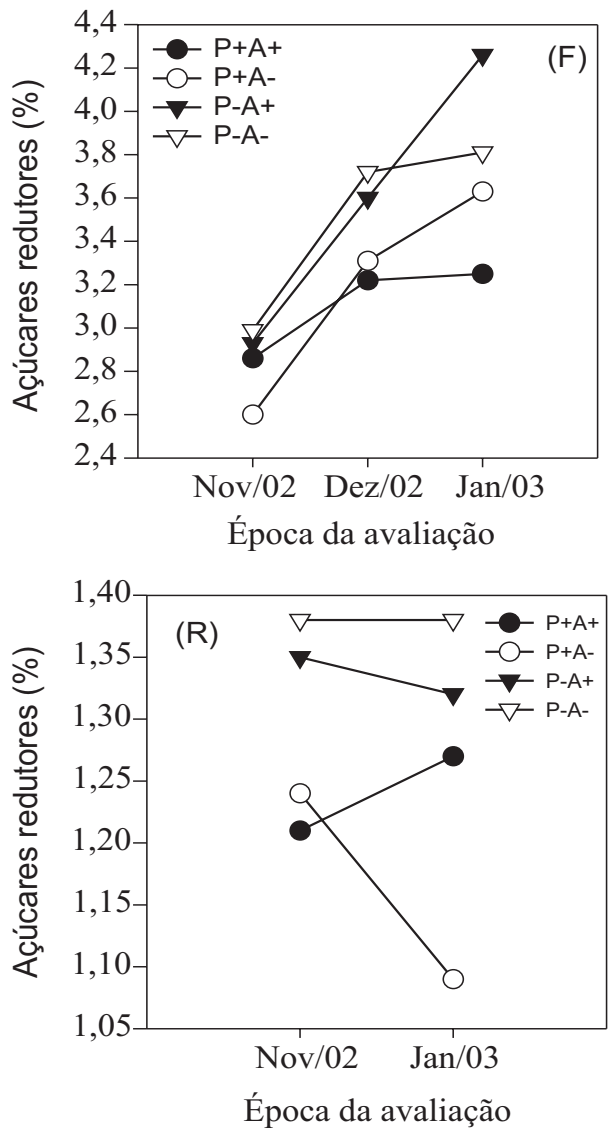
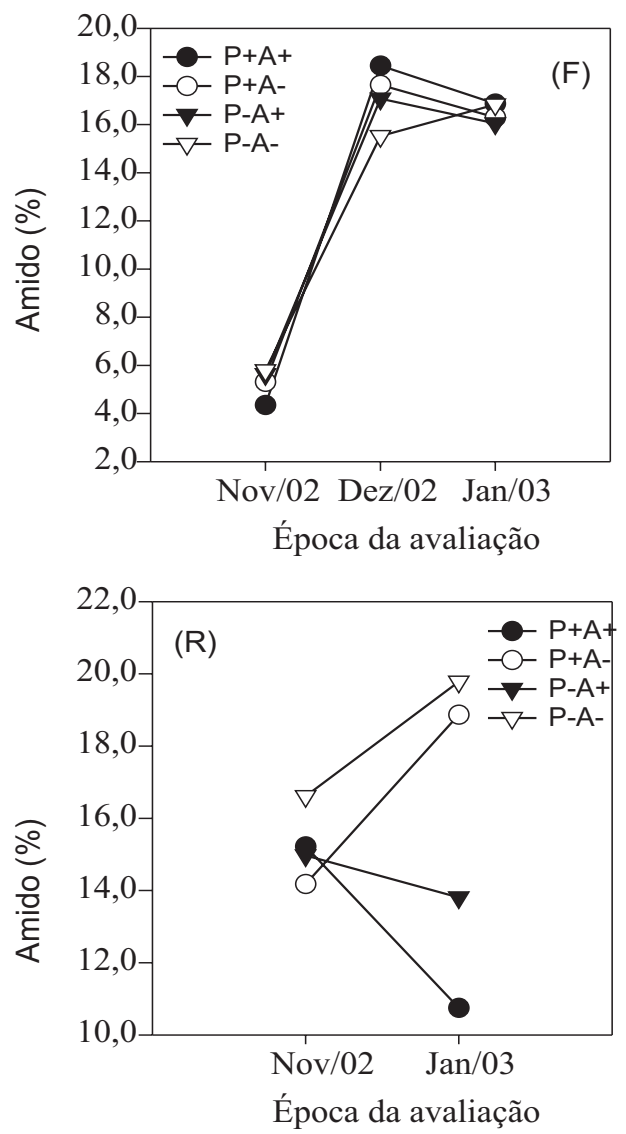

FIGURA 4 - Concentrações de carboidratos (AST, AR e AMIDO) nas folhas (F) e raízes (R) da tangerineira satsuma 'Owari’ mantidas em ambiente com temperaturas de $15^{\circ} \mathrm{C} / 8^{\circ} \mathrm{C}$ (dia/noite), tratadas com PBZ e aneladas (experimento 15/8). 
e, conseqüentemente, maior demanda para a utilização de reservas, principalmente de amido armazenado no sistema radicular. Resultados semelhantes foram obtidos por JONES et al. (1975) para a tangerineira 'Kinnow', que apresentou redução nas concentrações de amido no sistema radicular em função do alto florescimento das plantas.

\section{CONCLUSÃO}

Temperaturas baixas $\left(15 / 8{ }^{\circ} \mathrm{C}\right.$ dia/noite $)$ aumentaram o florescimento da satsuma 'Owari', enquanto a aplicação de paclobutrazol no solo e o anelamento no caule das plantas não influenciaram em seu florescimento.

\section{REFERÊNCIAS}

ACOSTA, J.F.; GONZALEZ, J.; RODRIGUEZ, R.; LEON, W. Effect of growth regulator applications on the juvenile period of Valencia oranges (Citrus sinensis). Centro Agrícola, Santa Clara, v.21, p.51$56,1994$.

DAVENPORT, T.L. Citrus flowering. Horticultural Reviews, New York, v.12, p.349-408, 1990.

DAVENPORT, T.L. Efficiency of paclobutrazol on tropical fruit growth. Proceedings Plant Growth Regulation Society of America, v. 13, p.242-243, 1987a.

DAVENPORT, T.L. Oportunities for tropical crop growth regulation. Proceedings Plant Growth Regulation Society of America, v. 13, p.406-409, 1987b.

DAVIES, F. S.; ALBRIGO, L. G. Citrus. Wallingford, UK: CAB International, 1994. 254p.

DELGADO, R.; RODRIGUEZ, R.; CASAMAYOR, R. Empleo de paclobutrazol em plantas de lima persa sobre naranjo trifoliado 'Rubdoux' a altas densidades. Agrícola Vergel, p.121-125, 1995.

DELGADO, R.R.; CASAMAYOR, J.L.; RODRIGUEZ, P.; CRUZ, R. F. R. Paclobutrazol effects on oranges under tropical conditions. Acta Horticulturae, Wazenizen, v.179, p.537-543, 1986.

FLETCHER, R.A.; GILLEY, A.; SANKLA, N.; DAVIS, T.D. Triazoles as plant growth regulators and stress protectants. Horticultural Reviews, New York, v.24, p.55-138, 2000.

GARCÍA-LUIS, A.; KANDUSER, M. Changes in dormancy and sensitivity to vernalization in axillary buds of Satsuma mandarine examined in vitro during the annual cicle. Annals of Botany, London, v. 76, p. 451-455, 1995 .

GOLDSCHMIDT, E.E. AND MONSELISE, S.P. Hormonal control of flowering in citrus and some other woody perennials. In: J.D. Carr (Ed.) Plant growth substances. Berlin: Springer, 1970. p. 758-765.

GRAEBE, J.E. Gibberellin biosynthesis and control. Annual Review of Plant Physiology, Palo Alto, v. 38, p.419-465, 1987.

HARTY, A.R.; STADEN, V.J. Paclobutrazol and temperature effects on lemon. In: INTERNATIONAL CITRUS CONGRESS, 6., Tel Aviv, 1988. Proceedings... International Society of Citriculture, 1988. p.343353.
IWAHORI, S.; TOMINAGA, S. Increase in first-flush flowering of 'Meiwa' kunquat, Fortunella crassifolia Swingle, trees by paclobutrazol. Scientia Horticulturae, Amsterdam, v.28, p.347-353, 1986.

JONES, W. W.; EMBLETON, T. W.; COGGINS Jr., C. W. Starch content of roots of 'Kinnow' mandarin trees bearing fruit in alternate years. Hortscience, Alexandria, v.10, p.514, 1975.

McCREDY, R. M.; GUGGOLZ, J.; SILVIERA, V.; OWENS, H. S. Determination of starch and amylose in vegetables: aplication to peas. Analytical Chemistry., Washington, v. 22, n. 9, p. 1156-1158, 1950.

MEHOUACHI, J.; TADEO, F.R.; ZARAGOZA, S.; PRIMO MILLO, E.; TALON, M. Effects of gibberellic acid and paclobutrazol on growth and carbohidrate accumulation in shoots and roots of citrus rootstock seedlings. Journal of Horticultural Science, Ashford, v.71, p.747-754, 1996.

MOSS, G. I. Influence of temperature and photoperiod on flower induction and inflorescence development in sweet orange (Citrus sinensis L. Osbeck). Journal of Horticultural Science, Ashford, v.44, p.311-314, 1969.

OGATA, T.; UEDA, Y.; SHIOZAKI, S.; HORIUCHI, S.; KAWASE, K. Effects of gibberellin synthesis inhibitors on flower settings of Satsuma mandarin. Journal of the Japanese Society for Horticultural Science, Sakyo-Ru, v.64, p.251-259, 1995.

OKUDA, H.; KIHARA, T.; IWAGAKI, I. Effects of paclobutrazol application to soil at the begining of maturation on sprouting, shoot growth, flowering and carbohidrate contents in roots and leaves of Satsuma mandarine. Journal of Horticultural Science, Ashford, v.71, p.785-789, 1996.

PARK, J. T.; JOHNSON, M. J. A submicrodetermination of glucose. Journal of Biological Chemistry. Bethesda, v. 181, p.149-151, 1949.

RADEMACHER, W. Bioregulation of crop plants with inhibitors of gibberellin byosinthesis. Proceedings Plant Growth Regulation of Society America, v.24, p.27-31, 1997.

SALISBURY, B.; ROSS, W. Plant Physiology. Belmont: Wadsworth Publisihng Company, 1991. 682p.

SNOWBALL, A.M.; WARRINGTON, I. J.; HALLIGAN, E.A.; MULLINS, M.G. Phase -change in citrus- the effects of main stem node number, branch habit and paclobutrazol apllication on flowering in citrus seedlings. Journal of Horticultural Science, Ashford, v.69, p.149$160,1994$.

SPIEGEL-ROY, P.; GOLDSCHMIDT, E. E. Reproductive physiology: flowering and fruiting. In:__ Biology of citrus. Cambridge: Cambridge University Press, 1996. p. 70-125.

YAMASHITA, K.; KITAZONO, K.; IWASAKI, S. Flower bud differentiation of Satsuma mandarin as promoted by soil drenching treatment with IAA, BA or paclobutrazol solution. Journal of the Japanese Society for Horticultural Science, Sakyo-Ru, v.66 p.67$76,1997$. 